\title{
Stochastic Volatility with Reset at Jumps
}

\author{
Jun Pan*
}

December 18, 1997

\begin{abstract}
This paper presents a model for asset returns incorporating both stochastic volatility and jump effects. The return process is driven by two types of randomness: small random shocks and large jumps. The stochastic volatility process is affected by both types of randomness in returns. Specifically, in the absence of large jumps, volatility is driven by the small random shocks in returns through a $\operatorname{GARCH}(1,1)$ model, while the occurrence of a jump event breaks the persistence in the volatility process, and resets it to an unknown deterministic level. Model estimation is performed on daily returns of S\&P 500 index using the maximum-likelihood method. The empirical results are discussed.
\end{abstract}

Recently, there has been fair amount of work in the asset pricing literature that studies models with both jump and stochastic volatility dynamics. For

${ }^{*}$ Graduate School of Business, Stanford, CA 94305. junpan@stanford.edu. I am grateful for extensive discussions with Darrell Duffie and Kenneth Singleton. I would also like to thank Geert Bekaert for helpful comments. 
example, in the discrete-time setting, Jorion [1989] employs a jump model with $\mathrm{ARCH}(1)^{1}$ to test equity returns and exchange rates, and Bekaert and Gray [1996] use a jump with $\operatorname{GARCH}(1,1)$ model to study the target zones and exchange rates. In the continuous setting, jump-diffusion models with stochastic volatility can be found in Bates [1997] and Bakshi, Cao, and Chen [1997], among others.

Although existing empirical work has clearly shown the importance of characterizing the dynamics of both jumps and stochastic volatility in asset returns, it still remains an open question as how these two dynamics interact with each other. To be more specific, one important question is: how does a jump in return affect the dynamics of the stochastic volatility process? In Jorion [1989], the volatility dynamics are affected only through the mixture of random shock and jumps, and as a consequence, this volatility process can not differentiate large jumps from small random movements. Moreover, because of the ARCH dynamics, the volatility process can "overshoot" to an unreasonably high level due to jumps in returns. Bekaert and Gray [1996] employ a "pressure relief" mechanism to capture the phenomenon that certain types of jumps in exchange rates break up the persistence in the volatility process. However, in their paper, the arrival of such "pressure reliefs" is given exogenously. Bates [1997] and Bakshi, Cao, and Chen [1997] model the stochastic volatility with an autonomous process, which is independent of the return process, hence one can not rely on these models to study the jump effect on a stochastic volatility process.

In this paper, we develop a simple model for asset return that incorporates

\footnotetext{
${ }^{1}$ For details on ARCH and GARCH models, see Engle [1982] and Bollerslev [1986].
} 
both the jump and stochastic volatility dynamics: the small (marginal) movements in returns are driven by a sequence of random shocks with stochastic volatility, whereas the arrival of jumps is dictated by Bernoulli trials with a normally distributed jump size. To study explicitly the affect of return jumps on the dynamics of stochastic volatility, we introduce a volatility model with "resets" at jumps. Specifically, in the absence of a jump, the volatility is driven by the small random shock, which can be associated with marginal movements in returns, through a $\operatorname{GARCH}(1,1)$ dynamic, while the arrival of a jump in returns "resets" the volatility level to an unknown deterministic level. This reset mechanism allows the volatility process to "refresh its memory" and breaks its persistence pattern. Such a "pressure relief" phenomenon in the volatility process, caused by realizations of surprises, can be observed in many markets, for example, in the exchange-rate market. ${ }^{2}$

Given that this model accommodates three random sources (marginal movement, jump arrival, and jump size), we can no longer exactly invert the sample path of the volatility process from observations of asset returns. This problem with un-observable variables can potentially make maximumlikelihood estimation infeasible. However, because of the volatility reset feature incorporated in this model, we are able to back out a conditional volatility tree whose dimension grows linearly with the number of periods. As a consequence, this model specification has the advantage of moderate demand in model estimation using the maximum-likelihood method.

Finally, this model with volatility reset nests $\operatorname{GARCH}(1,1)$, and jump with constant volatility models.

\footnotetext{
${ }^{2}$ See Bekaert and Gray [1996] for details.
} 
The rest of the paper is organized as follows. In Section I, we specify the model, and sketch the calculation of the sample likelihood function. In Section II, we apply this model to the S\&P 500 daily returns, and discuss the empirical results. In Section III, we discuss possible extensions of the model. In Section IV, we conclude the paper.

\section{Model Specification and Implementation}

\section{A. Model Specification}

Under a discrete-time setting, let $\epsilon=\left\{\epsilon_{t}: t=1,2, \ldots\right\}$ be a sequence of $i . i . d$. random variables with standard normal distribution, $J=\left\{J_{t}: t=1,2, \ldots\right\}$ be Bernoulli trials with success probability $p,{ }^{3}$ and $Z=\left\{Z_{t}: t=1,2, \ldots\right\}$ be a sequence of $i . i . d$. random variables normally distributed with mean $\mu_{Z}$ and variance $\sigma_{Z}^{2} \cdot\{\epsilon\},\{J\}$, and $\{Z\}$ are mutually independent. We model the return process by an $\mathbb{R}$-valued process $y$ such that

$$
y_{t+1}=\mu+\sqrt{h_{t}} \epsilon_{t+1}+Z_{t+1} J_{t+1},
$$

where $\mu \in \mathbb{R}$, and where the $\mathbb{R}_{+}$-valued process $h$ is defined by

$$
h_{t}=\bar{h} J_{t}+g\left(y_{t}, h_{t-1}\right)\left(1-J_{t}\right), \quad h_{0} \in \mathbb{R}_{+},
$$

where the GARCH function $g: \mathbb{R} \times \mathbb{R}_{+} \rightarrow \mathbb{R}_{+}$is defined by

$$
g(y, h)=a_{0}+a_{1}(y-\mu)^{2}+a_{2} h,
$$

\footnotetext{
${ }^{3}$ That is, for a fixed time $t, J_{t}=1$ with probability $p$, and $J_{t}=0$ with probability $1-p$. For any times $t \neq s, J_{t}$ and $J_{s}$ are independent.
} 
where $a_{0}, a_{1}$, and $a_{2}$ are in $\mathbb{R}_{+}$.

In the above model, the time- $t$ jump arrival is dictated by $J_{t}$, while the jump size in return is controlled by $Z_{t}$. At time $t$, the marginal movement in return is modeled by $\epsilon_{t}$ with a stochastic volatility $\sqrt{h_{t-1}}$. Both $J$ and $\epsilon$ contribute to the dynamics of $h$. Specifically, conditioning on a jump event at time $t$, that is $J_{t}=1, h_{t}$ is reset to a constant level $\bar{h}$, while, in the absence of a jump at time $t, h_{t}$ follows the usual $\operatorname{GARCH}(1,1)$ process.

\section{B. Model Implementation}

The empirical methodology we adopt for this model estimation is maximum likelihood. For a general model with stochastic volatility, implementation of the likelihood function could be computationally infeasible because of the unobservable variable $h_{t}{ }^{4}$ In this paper, however, the special volatility model with reset at jumps allows us to back out the conditional distribution of $h_{t}$ explicitly, and requires moderate computational demand to calculate the likelihood function.

For a time series of $T$ observations, we write the sample likelihood function

$$
f\left(y_{T}, y_{T-1}, \cdots, y_{2}, y_{1}\right)=\prod_{t=0}^{T-1} f\left(y_{t+1} \mid \mathcal{I}_{t}\right)
$$

where $\mathcal{I}_{t}=\left\{y_{1}, y_{2}, \ldots, y_{t}\right\}$, and $\mathcal{I}_{0}=\emptyset$.

Let $\tau$, the random time since last jump, be defined by

$$
\tau_{t}=\min \left\{t-s: s \leq t, J_{s}=1\right\} .
$$

${ }^{4}$ See, for example, Jacquier, Polson, and Rossi [1994], and references therein for model estimation techniques under a more general stochastic volatility structure. 
Noting that $\tau_{t} \in\{0,1, \ldots, t\}$, we write

$$
f\left(y_{t+1} \mid \mathcal{I}_{t}\right)=\sum_{i=0}^{t} f\left(y_{t+1} \mid \mathcal{I}_{t}, \tau_{t}=i\right) P\left(\tau_{t}=i \mid \mathcal{I}_{t}\right) .
$$

Conditional on the outcome of $J_{t+1}$, we have

$$
\begin{aligned}
f\left(y_{t+1} \mid \mathcal{I}_{t}, \tau_{t}=i\right)= & p f\left(y_{t+1} \mid \mathcal{I}_{t}, \tau_{t}=i, J_{t+1}=1\right) \\
& +(1-p) f\left(y_{t+1} \mid \mathcal{I}_{t}, \tau_{t}=i, J_{t+1}=0\right)
\end{aligned}
$$

where $p$ is the success rate of the Bernoulli trial.

Using the dynamics of $h$ defined in (2), we know that, at time $t=$ $1,2, \ldots, T, h_{t}$ depends entirely on $\mathcal{I}_{t}$ and $\tau_{t}$. Specifically, we denote $H_{t}(i)$ to be the outcome of $h_{t}$ conditional on the event $\left\{\mathcal{I}_{t}, \tau_{t}=i\right\}$, and we immediately have $H_{0}(0)=h_{0}$, and $H_{t}(0)=\bar{h}$ for any $t=1,2, \ldots, T$. Moreover, for $i=$ $1,2, \ldots, t, H_{t}(i)$ can be calculated recursively by $H_{t}(i)=g\left(y_{t}, H_{t-1}(i-1)\right)$, for $i=1, \ldots, t$.

It then follows that

$$
\begin{aligned}
& f\left(y_{t+1} \mid \mathcal{I}_{t}, \tau_{t}=i, J_{t+1}=1\right)=\varphi^{A}\left(y_{t+1}, H_{t}(i)\right) \\
& f\left(y_{t+1} \mid \mathcal{I}_{t}, \tau_{t}=i, J_{t+1}=0\right)=\varphi^{B}\left(y_{t+1}, H_{t}(i)\right),
\end{aligned}
$$

where $\varphi^{A}: \mathbb{R} \times \mathbb{R}_{+} \rightarrow \mathbb{R}_{+}$, and $\varphi^{B}: \mathbb{R} \times \mathbb{R}_{+} \rightarrow \mathbb{R}_{+}$are defined by

$$
\begin{aligned}
\varphi^{A}(x, b) & =\frac{1}{\sqrt{2 \pi\left(b+\sigma_{Z}^{2}\right)}} \exp \left(-\frac{\left(x-\mu-\mu_{Z}\right)^{2}}{2\left(b+\sigma_{Z}^{2}\right)}\right) \\
\varphi^{B}(x, b) & =\frac{1}{\sqrt{2 \pi b}} \exp \left(-\frac{(x-\mu)^{2}}{2 b}\right)
\end{aligned}
$$

As it is illustrated in Figure 1, we may intuitively think of

$$
\left[H_{t}(0), H_{t}(1), \ldots, H_{t}(t)\right]
$$


as the time- $t$ branch of a volatility tree inverted back from observations of returns up to time $t$. Moreover, given $\mathcal{I}_{t}$, the probability that $h_{t}$ ends up at the $i$-th node with value $H_{t}(i)$ is simply $\pi_{t}(i)=P\left(\tau_{t}=i \mid \mathcal{I}_{t}\right)$. We next derive a recursive formulation to calculate $\pi_{t}(i)$. Let $\pi_{0}(0)=1$, and for any $t \geq 1$ and $i=1, \ldots, t$, we have

$$
\begin{aligned}
\pi_{t}(i) & =P\left(\tau_{t}=i, \tau_{t-1}=i-1 \mid \mathcal{I}_{t}\right) \\
& =P\left(\tau_{t}=i \mid \mathcal{I}_{t}, \tau_{t-1}=i-1\right) P\left(\tau_{t-1}=i-1 \mid \mathcal{I}_{t}\right) \\
& =P\left(J_{t}=0 \mid \mathcal{I}_{t}\right) P\left(\tau_{t-1}=i-1 \mid \mathcal{I}_{t}\right),
\end{aligned}
$$

where

$$
\begin{aligned}
& P\left(J_{t}=0 \mid \mathcal{I}_{t}\right)=(1-p) \frac{f\left(y_{t} \mid \mathcal{I}_{t-1}, J_{t}=0\right)}{f\left(y_{t} \mid \mathcal{I}_{t-1}\right)} \\
& P\left(\tau_{t-1}=i-1 \mid \mathcal{I}_{t}\right)=\pi_{t-1}(i-1) \frac{f\left(y_{t} \mid \mathcal{I}_{t}, \tau_{t-1}=i-1\right)}{f\left(y_{t} \mid \mathcal{I}_{t-1}\right)}
\end{aligned}
$$

where

$$
\begin{aligned}
& f\left(y_{t} \mid \mathcal{I}_{t-1}, J_{t}=0\right)=\sum_{i=1}^{t} \varphi^{B}\left(y_{t}, H_{t-1}(i-1)\right) \pi_{t-1}(i-1) \\
& f\left(y_{t} \mid \mathcal{I}_{t}, \tau_{t-1}=i-1\right)=p \varphi^{A}\left(y_{t}, H_{t-1}(i-1)\right)+(1-p) \varphi^{B}\left(y_{t}, H_{t-1}(i-1)\right) \\
& f\left(y_{t} \mid \mathcal{I}_{t-1}\right)=\sum_{i=1}^{t} f\left(y_{t} \mid \mathcal{I}_{t}, \tau_{t-1}=i-1\right) \pi_{t-1}(i-1) .
\end{aligned}
$$

And finally we obtain $\pi_{t}(0)$ from $\pi_{t}(0)=1-\sum_{i=1}^{t} \pi_{t}(i)$, for $t=1, \ldots, T$.

\section{Application to S\&P 500 Daily Returns}

As an application, we estimate the parameters of the model specified in (1) and (2) ("Full Model") by maximum likelihood using daily returns of the 
S\&P 500 composite index from January 1986 to January 1997. The time series has a total of of 2,870 observations.

In addition to estimating the "Full Model", we also estimate two nested models: (1) GARCH(1,1), a special case of the "Full Model" with $J_{t}=0$ (or $p=0$ ); (2) Jump with constant volatility, another special case of the "Full Model" with $a_{0}=\bar{h}, a_{1}=0$, and $a_{2}=0$. The outcome of the parameter estimates and relevant statistics, for all three models, is reported in Table I. A likelihood ratio test soundly rejects the model of jump with constant volatility against the "Full Model". Because of the missing "nuisance" parameters, the test of $\operatorname{GARCH}(1,1)$ against the "Full Model" is non-standard. Specifically, the large sample distribution of the likelihood ratio statistics $(L R)$ is unknown. A bound of the probability distribution of $L R$ could be obtained using a grid method such as that in Hansen [1992], but we omit this test and simply point out the significant improvement of the log-likelihood value.

Using the "Full Model" estimates reported in Table I, we may summarized the model-dependent characteristics of the daily movement of the S\&P 500 index as following:

- Base of the jump probability of $1.24 \%$, we can calculate that significant "surprise" price movement happens, on average, about three times a year. These small-probability jump events are, in expectation, $1.83 \%$ downward, with a standard deviation of $4.9 \% .^{5}$

- There are high persistence in the volatility process : the first order autocorrelation of the volatility process is $a_{1}+a_{2}=0.9857$ with a standard

\footnotetext{
${ }^{5}$ Given these estimates, a $20 \%$ downward jump like that in the 1987 Crash will take more than ten thousand years to happen once.
} 
error of 0.0047 , conditioning on no jump events. The unconditional auto-correlation could be calculated as $(1-p)\left(a_{1}+a_{2}\right)=0.9736$, with a standard error of 0.0056 .

- Right after each jump, the stochastic volatility starts "afresh" from a relatively higher level $(\sqrt{h}=16.6 \%)$ than the steady-state average level $\left(\sqrt{E\left(h_{t}\right)}=13.0 \%\right)$. As the mean of jump returns is estimated to be $-1.83 \%$, the effect of negative correlation between downward price movement and high levels of volatility is captured, with $\operatorname{cov}\left(y_{t}, h_{t}\right)=$ $p \mu_{Z}\left(\bar{h}-E\left(h_{t}\right)\right)$. From the model estimates, $\operatorname{corr}\left(y_{t}, h_{t}\right)=-0.0319$, with a standard error of 0.0174 .

The rest of the section is organized as following: In section A, we discuss the jump effect in daily returns picked up by our model and the model of jump with constant volatility, and stress the point why stochastic volatility is essential in modeling jump effect. In section B, we compare the stochastic volatility process implied by our model with that implied by $\operatorname{GARCH}(1,1)$, and stress the point why stochastic volatility alone is not adequate in han-

dling time series with jump effect. And finally, in section C, we study the population moments implied by all of the three models, and compare them with the sample moments to understand how well each model characterizes the distribution of the time series.

\section{A. Model Characterization of Jumps}

In order to study how well the model characterizes jumps, Figure 2 plots $\mathcal{P}\left(J_{t} \mid \mathcal{I}_{t}\right)$, the probability of jump at time $t$ updated by observations of re- 
turns up to time $t$. The top panel is obtained using the "Full Model", while the bottom panel is obtained using the model of jump with constant volatility. From this result, we notice that the jump model with constant tends to overestimates the probability of jump events. From the model estimates in Table I, we can also see that the model of jump with constant volatility picks smaller jumps with a higher frequency. This could be explained by the inflexibility of the volatility structure, which results in mistaking high volatility levels as small jumps.

\section{B. Model Characterization of Stochastic Volatility}

Figure 3 combines $\sqrt{h_{t}}$ inferred from $\operatorname{GARCH}(1,1)$ and $\sqrt{E\left(h_{t} \mid \mathcal{I}_{t}\right)}$ implied by the "Full Model" on the same plot, and shows the fact that GARCH(1,1) "overshoots" the level of volatility in the event of large price movements. For example, the volatility level jumps to an unreasonably high of $112.6 \%$ in the 1987 Crash. This is a direct consequence of the mis-specification of the GARCH model, which fails to incorporate jump effects. As a result, a high level of $\left|y_{t}-\mu\right|$ accompanying a jump event will enter the volatility process through $\sigma_{t}^{2} \sim a_{1}\left(y_{t}-\mu\right)^{2}$, and cause a high volatility level to explain the jump event. For the same reason, $a_{1}$ is estimated to be significantly larger in the GARCH $(1,1)$ model than the full model, and the opposite case for $a_{2}$.

Figure 4 plots the conditional expectation of volatility updated by observations, $E\left(h_{t} \mid \mathcal{I}_{t}\right)$, and Figure 5 plots the conditional variance of volatility updated by observations, $\operatorname{var}\left(h_{t} \mid \mathcal{I}_{t}\right)$. The highest level $(\sqrt{0.07}=26 \%)$ of volatility occurs around the 87-Crash, when the highest conditional variance of volatility also occurs, at the level of $0.03 \%$. The variation range of the 
volatility process and the variance of the volatility depicted in Figure 4 and 5 seems, subjectively, to be reasonable estimates for the volatility process and its variance.

\section{Model Characterization of Moments}

In order to examine how well the model characterizes the distribution of the time series in terms of distribution, we study the moments of S\&P 500 daily-return distribution implied by the model estimates, and compare them with the sample moments. The point estimates, as well as the estimated standard errors, are listed in Table II. In obtaining the standard errors for the sample estimates, we use a GMM-style approach for the four exactlyidentified moments. The covariance estimator of the sample moments is obtained using the Newey-West method with 5 lags. ${ }^{6}$

Skewness is a measure of the degree to which positive deviations from the mean are larger than the negative deviations from the mean. A negative skewness means that large negative returns are more likely than large positive returns. From Table II, judging only from the point estimates, we see that the population skewness basically captures the negativity in sample skewness, however, the magnitude is underestimated. There are possibly two contributions to the skewness: one comes from jumps with negative mean; the other comes from negative correlation between the level of stochastic volatility $h_{t}$ and the random shock $\epsilon_{t}$, which is not incorporated in this model. However,

\footnotetext{
${ }^{6}$ See Hansen [1982] for large sample distributions of the GMM estimators, and Newey and West [1987] for the covariance estimator. For a more accessible exposure, see Hamilton [1994].
} 
at short time horizon, the skewness effect introduced by negative jumps is expected to be the more pronounced.

Kurtosis is a characteristic of tail-fatness. The large sample kurtosis of 113 is accompanied with a "huge" standard error of 54, resulting from infrequent observations of large moves. This is a direct consequence of the 1987 Crash, when the market moved over $20 \%$ in one day. After taking out this period from the sample, the kurtosis is less than 10. Also, if we measure, instead, weekly returns, the sample kurtosis reduces to 12. Judging from the point estimates of sample and population kurtosis, we can see that the population kurtosis implied by the model estimates severely underestimates the kurtosis of daily returns. Kurtosis estimated on a daily horizon is less precise than estimated kurtosis on a weekly or monthly horizon. A study of the term structure of kurtosis is not within the scope of this paper. We do acknowledge, however, the difficulty involved in estimating the high end of this kurtosis term structure.

\section{Model Extensions}

\section{A. Jump Clustering}

Equity returns of short time horizon usually exhibit "jump clustering," that is, large movements in returns tend to happen together within short time period. To model this effect, we may allow the time- $t$ success rate $p_{t}$ of the Bernoulli trial $J_{t}$ to depend on the history of jumps. Specifically, let $p_{t}=\gamma\left(\tau_{t-1}\right)$ be the probability that $J_{t}=1$, where $\tau$ is the random time since last jump defined in (4), and where $\gamma: \mathbb{N} \rightarrow[0,1]$. By allowing $p_{t}$ to be 
a function of the past jump history, we effectively model the phenomena of "jump clustering." Moreover, maximum-likelihood estimation is still feasible under this extension. Specifically, equation (6) in Section 1B becomes

$$
\begin{aligned}
f\left(y_{t+1} \mid \mathcal{I}_{t}, \tau_{t}=i\right)= & \gamma(i) f\left(y_{t+1} \mid \mathcal{I}_{t}, \tau_{t}=i, J_{t+1}=1\right) \\
& +(1-\operatorname{gamma}(i)) f\left(y_{t+1} \mid \mathcal{I}_{t}, \tau_{t}=i, J_{t+1}=0\right) .
\end{aligned}
$$

\section{B. Volatility Asymmetry}

We may capture the volatility asymmetry effect contributed by $\epsilon$ and $h$ by using an E-GARCH dynamics. Specifically,

$$
\ln \left(h_{t}\right)=\ln (\bar{h}) J_{t}+g^{*}\left(y_{t}, \ln \left(h_{t-1}\right)\right)\left(1-J_{t}\right), \quad h_{0} \in \mathbb{R}_{+},
$$

where $g^{*}: \mathbb{R} \times \mathbb{R} \rightarrow \mathbb{R}$ is defined by

$$
g^{*}(x, v)=a_{0}+a_{1} x+a_{2} x^{2}+a_{3} v,
$$

where $a_{0}, a_{1}, a_{2}$, and $a_{3}$ are in $\mathbb{R}$.

Conditional on no jumps, the conditional volatility is affected by the sign of $\epsilon$. Hence, we effectively introduce a correlation between $\epsilon$ and $h$. Moreover, the maximum-likelihood estimation approach is still feasible under this extension.

\section{Relax the "Aftermath"}

One over-restrictiveness of this model is that the volatility process is reset to a deterministic level $\bar{h}$ conditional on a jump event. While this particular reset mechanism provides with us a manageable conditional volatility tree, this 
"aftermath" can be overly restrictive. By allowing $\bar{h}$ to be a random variable can relax this restriction, however, likelihood estimation is not feasible under this extension.

\section{Conclusion}

A simple and easy to implement model for asset returns is developed to incorporate both jump and stochastic volatility dynamics. The contribution of this paper lies mainly in the particular dynamics of the volatility process, that is, a volatility process with reset at jumps. The motivation behind this model is that "small" and "large" returns affect volatility through different channels, and a jump event usually relieves the system from its "built-up" pressure, and "refreshes" its memory.

As an application, this model is applied to daily returns of the S\&P 500 composite index from 1986 to 1997. We find that both the jump effect and stochastic volatility are useful features in characterizing the stochastic behavior in market returns. The effect of "over-shooting" of volatility level or "over-jumping" in returns could be found if either one component is missing from the model. Specifically, $\operatorname{GARCH}(1,1)$ and jump with constant volatility models are both mis-specified. We also find, through model estimation, that in the absence of jump event, volatility is highly persistent; jumps are negative in mean, and after a jump event, volatility starts at a level that is higher than its steady state mean. From this, we capture the effect of volatility asymmetry, or negative correlation between price movements and the volatility level. 
Implications of this model estimation on option pricing could be summarized as follows. First, we document the jump effect in daily returns of the S\&P 500, and provide maximum-likelihood estimates for this jump model with stochastic volatility. Hence option pricing could be readily implemented after an appropriate risk-neutralization, and be compared with observed option prices. From the model estimation, we invert a volatility tree that is implied by the underlying price, and further research can be conducted to compare this implied historical volatility tree with the option-implied volatility tree.

The basic characteristics imposed by this model on the time series of returns are quite general, and this model could be carried over naturally for estimations in stock returns in other markets, as well as in foreign exchange returns. The maximum-likelihood estimation of this model is very easy to set up, and the computational demand is moderate. The model can be further relaxed to allow jump probability $p$ depending on the history of jumps. This can be a very important feature to model the "jump clustering" effect. This model can also be extended to incorporate volatility asymmetric between the random shock $\epsilon$ and conditional volatility, by introducing an E-GARCH dynamics. However, the relaxation of $\bar{h}$ from deterministic to random (possibly depend on the size of last surprise) will make the maximum-likelihood approach adopted in this paper infeasible. 


\section{References}

Bakshi, G., C. Cao, and Z. Chen (1997). Empirical Performance of Alternative Option Pricing Models. Journal of Finance. forthcoming.

Bates, D. (1997). Post-'87 Crash Fears in S\&P 500 Futures Options. Working paper, National Bureau of Economic Research.

Bekaert, G. and S. Gray (1996). Target Zone and Exchange Rates : An empirical Investigation. Working paper, Graduate School of Business, Stanford.

Bollerslev, T. (1986). Generalized Autoregressive Conditional Hetreroskedasticity. Journal of Econometrics 31, 307-327.

Engle, R. (1982). Autoregressive Conditional Heteroskedasticity with Estimates of The Variance of United Kingdom Inflation. Econometrica 50, 987-1008.

Hamilton, J. (1994). Time Series Analysis. Princeton University Press.

Hansen, B. (1992). The likelihood Ratio Test under Non-standard Conditions: Testing the Markov Switching model of GNP. Journal of Applied Econometrics 7, S61-S82.

Hansen, L. (1982). Large Sample Properties of Generalized Method of Moments Estimators. Econonmetrica 50, 1029-1054.

Jacquier, E., N. Polson, and P. Rossi (1994). Bayesian Analysis of Stochastic Volatility Models (with Discussion). Journal of Business 83 Economic Statistics 12, 371-417. 
Jorion, P. (1989). On Jump Processes in the Foreign Exchange and Stock Markets. Review of Financial Studies 4, 427-445.

Newey, W. and K. West (1987). A simple Positive Semi-Definite, Hetreroskedasticity and Autocorrelation Consistent Covariance Matrix. Econometrica 55, 703-708. 


\section{List of Figures}

1 Inverting the Volatility Tree from the Observed Data, Under the Full Model and GARCH(1,1) Model. . . . . . . . . . 20

$2 \mathcal{P}\left(J_{t}=1 \mid \mathcal{I}_{t}\right)$, Probability of jumps updated by observations of daily returns of S\&P 500 Composite Index from 1986 to 1997. The top panel is estimated using the full model, while the bottom panel is estimated using the model of jump with constant volatility. . . . . . . . . . . . 21

3 Conditional volatility $\sqrt{E\left(h_{t} \mid \mathcal{I}_{t}\right)}$, for daily returns of S\&P 500 composite index from 1986 to 1997. Estimated using the Full Model and GARCH(1,1) . . . . . . . . . . . . 22

4 Expectation of the stochastic volatility conditioning on observations, $E\left(h_{t} \mid \mathcal{I}_{t}\right)$, for daily returns of S\&P 500 composite index from 1986 to 1997. Estimated using the Full Model. . . 23

5 Expected variance of the stochastic volatility conditioning on observations, $\operatorname{var}\left(h_{t} \mid \mathcal{I}_{t}\right)$, for daily returns of S\&P 500 composite index from 1986 to 1997. Estimated using the Full Model. . 24 

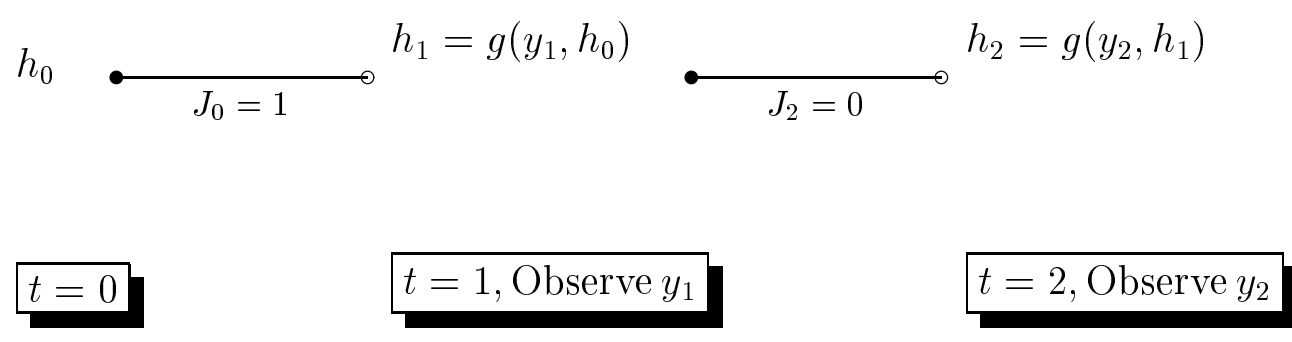

(a) Volatility Structure Under $\operatorname{GARCH}(1,1)$, a Special Case of Full Model with $J_{t} \equiv 0$.

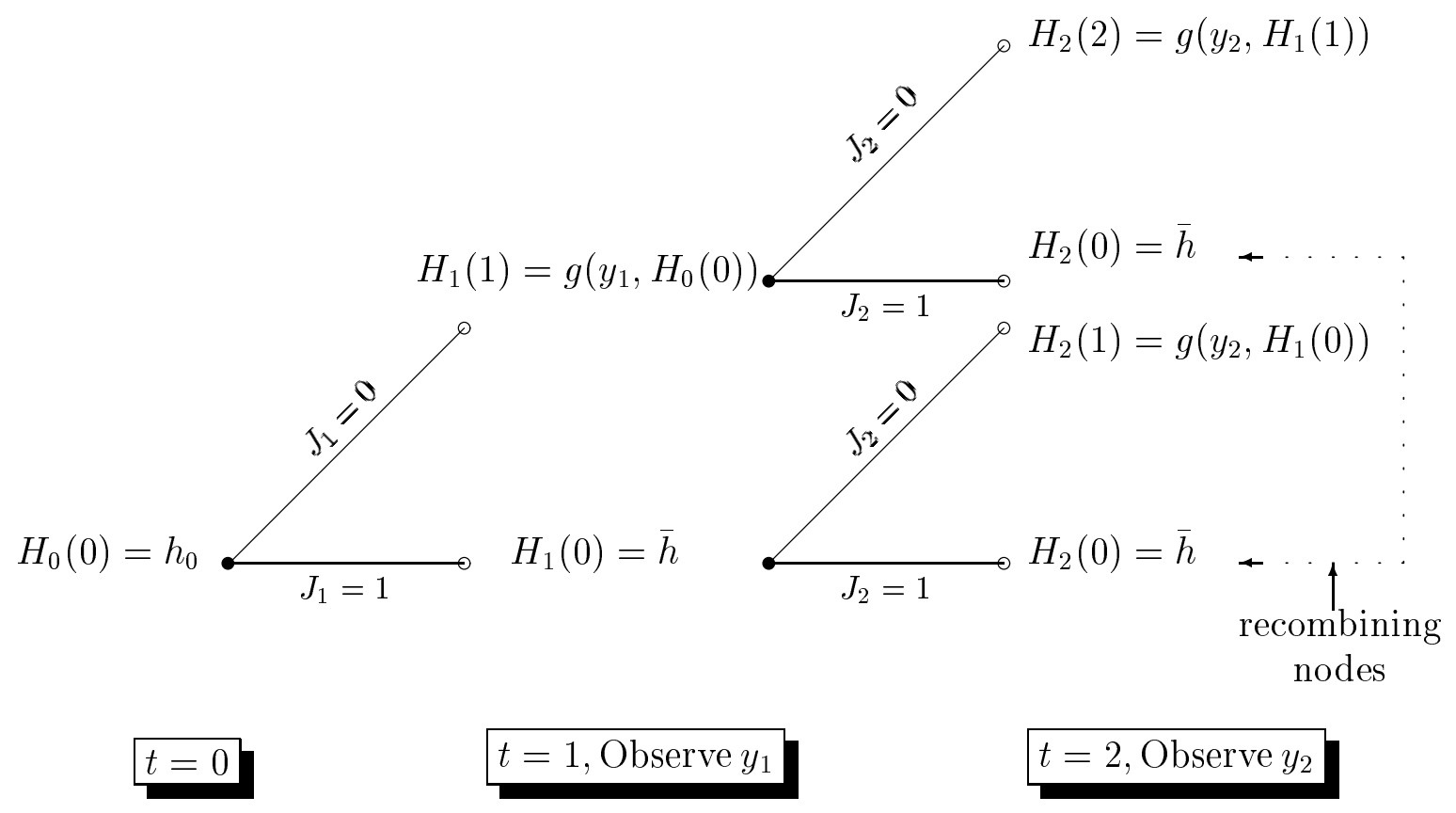

(b) Volatility Structure Under Full Model, Specified in (1) and (2).

Figure 1: Inverting the Volatility Tree from the Observed Data, Under the Full Model and GARCH(1,1) Model. 

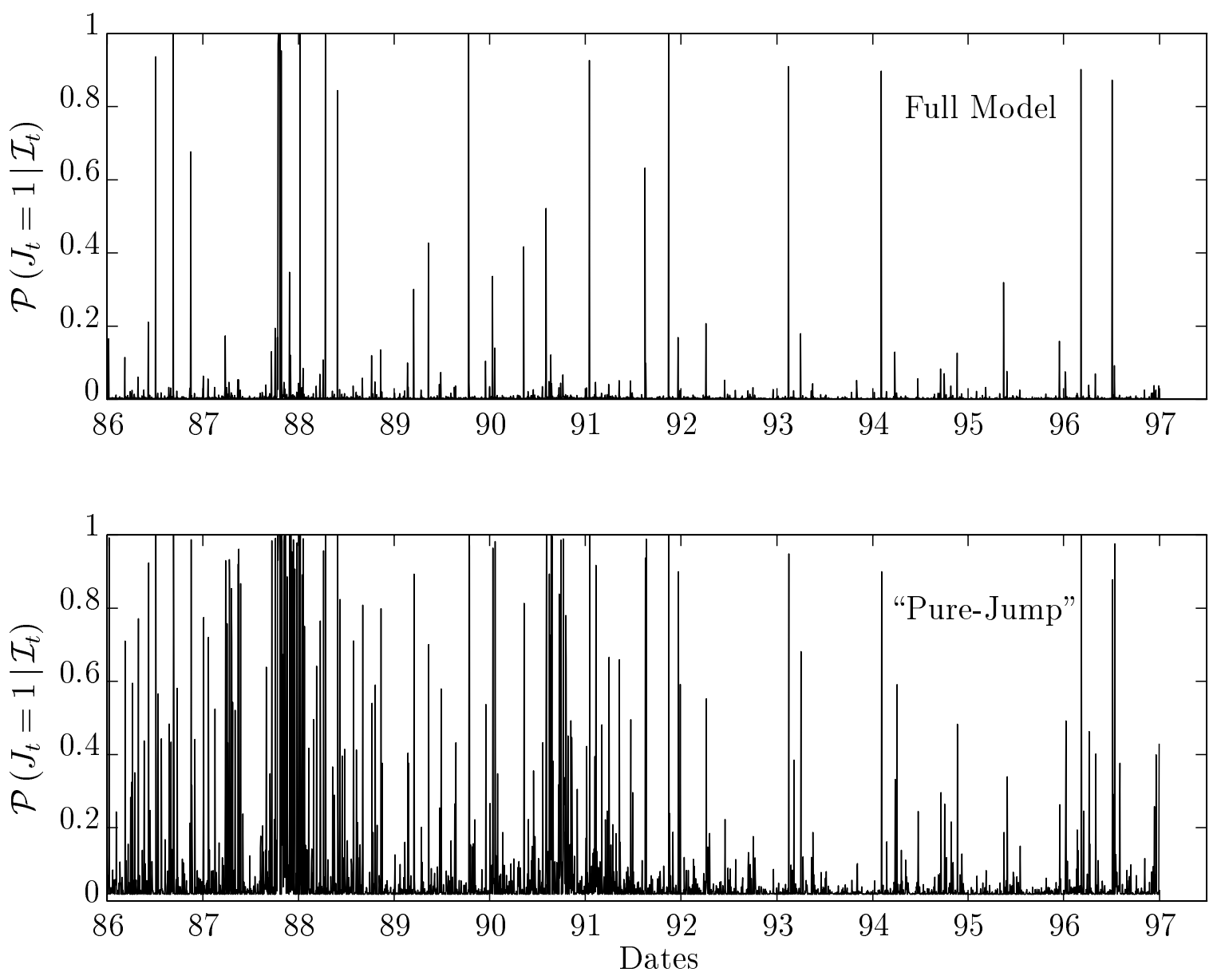

Figure 2: $\mathcal{P}\left(J_{t}=1 \mid \mathcal{I}_{t}\right)$, Probability of jumps updated by observations of daily returns of S\&P 500 Composite Index from 1986 to 1997. The top panel is estimated using the full model, while the bottom panel is estimated using the model of jump with constant volatility. 


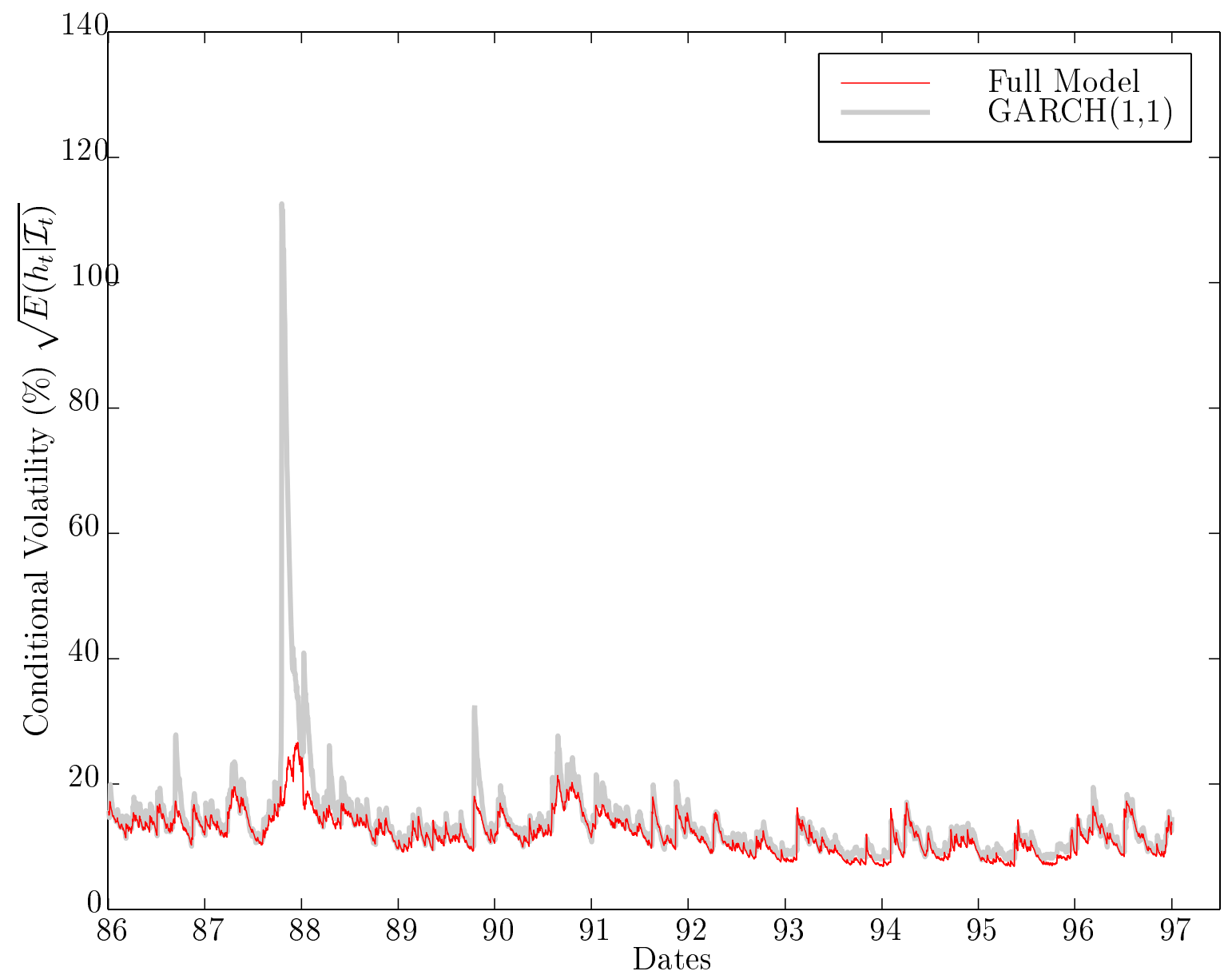

Figure 3: Conditional volatility $\sqrt{E\left(h_{t} \mid \mathcal{I}_{t}\right)}$, for daily returns of S\&P 500 composite index from 1986 to 1997. Estimated using the Full Model and $\operatorname{GARCH}(1,1)$. 


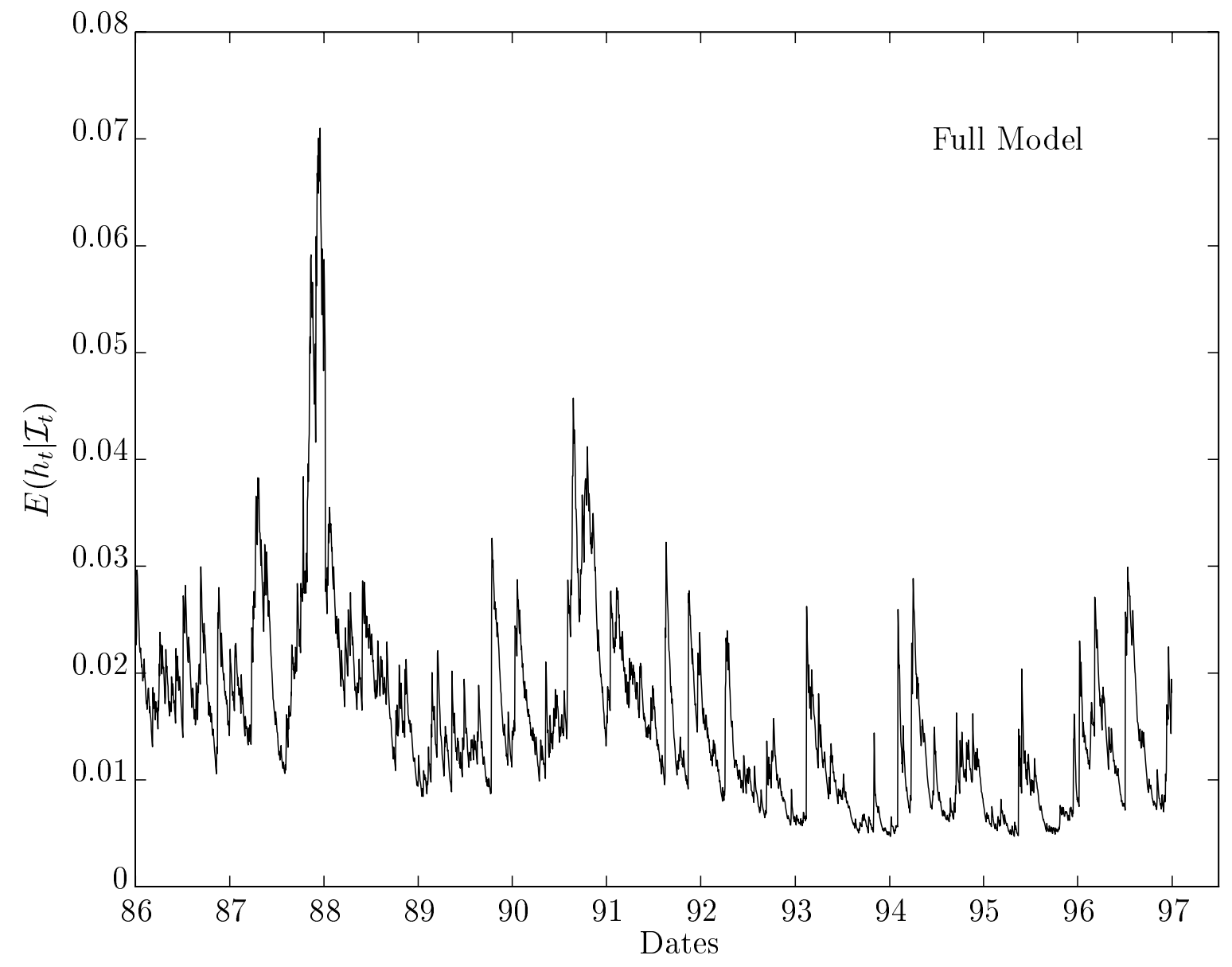

Figure 4: Expectation of the stochastic volatility conditioning on observations, $E\left(h_{t} \mid \mathcal{I}_{t}\right)$, for daily returns of S\&P 500 composite index from 1986 to 1997. Estimated using the Full Model. 


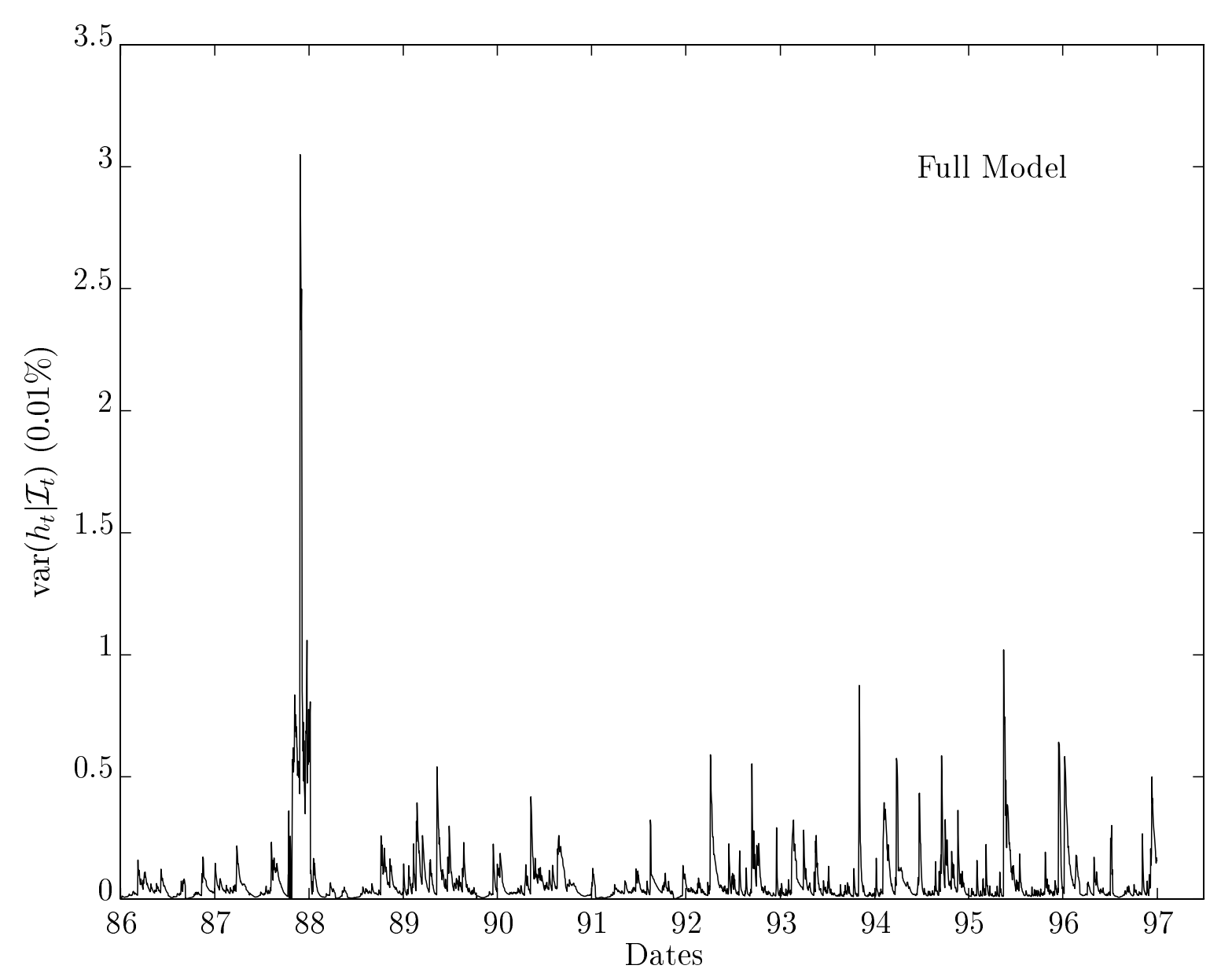

Figure 5: Expected variance of the stochastic volatility conditioning on observations, $\operatorname{var}\left(h_{t} \mid \mathcal{I}_{t}\right)$, for daily returns of S\&P 500 composite index from 1986 to 1997. Estimated using the Full Model. 


\section{List of Tables}

I Parameter estimates and related statistics for the jump-diffusion model with stochastic volatility and two of its nested models. . 26

II Estimates of the first four moments of daily returns of the S\&P 500 Composite Index from January 1986 to January 1997, with a total of 2870 observations $\ldots \ldots \ldots . \ldots 27$ 


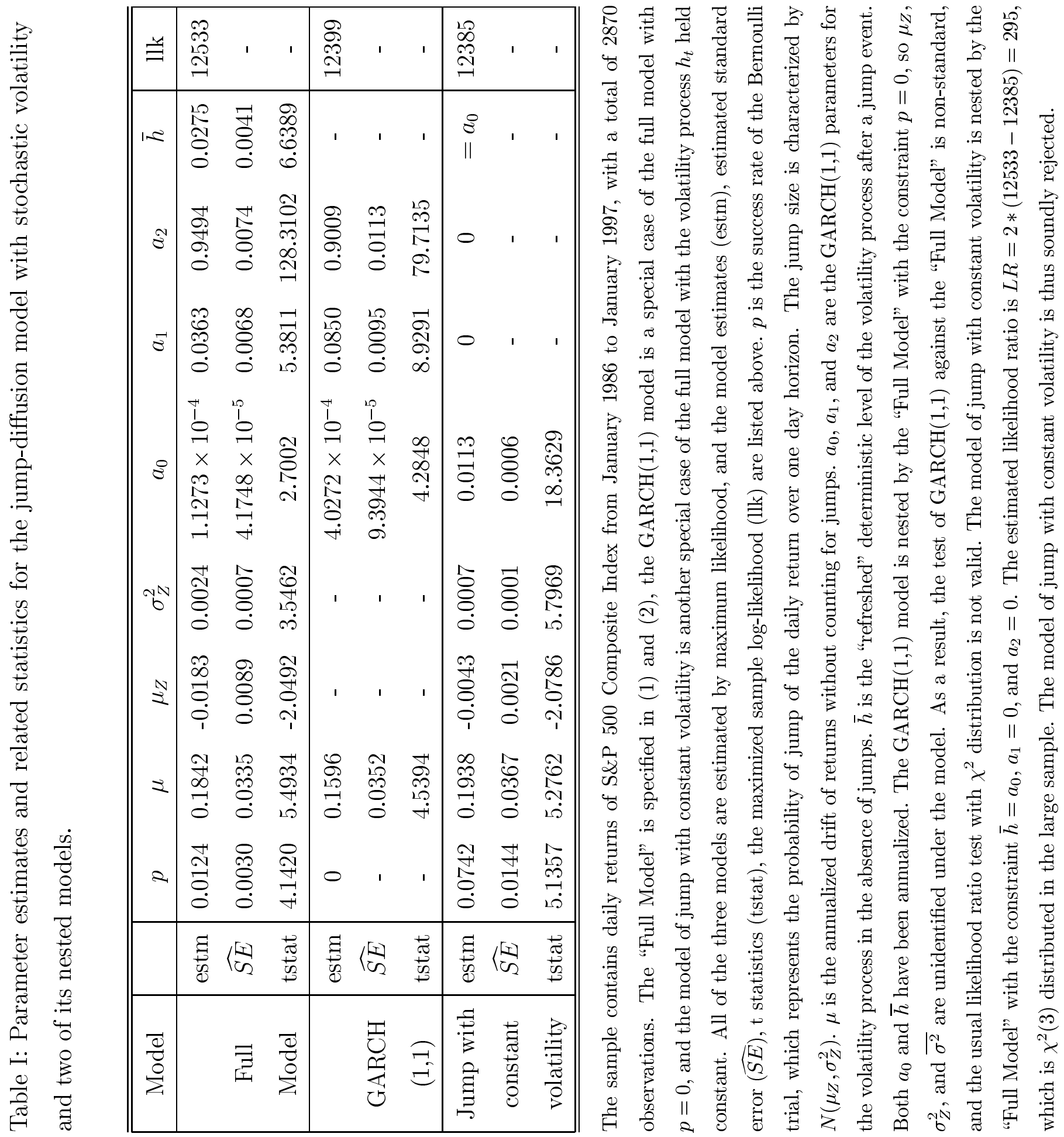


Table II: Estimates of the first four moments of daily returns of the S\&P 500 Composite Index from January 1986 to January 1997, with a total of 2870 observations

\begin{tabular}{|c|cccc|}
\hline \hline & mean & std & skew & kurtosis \\
\hline $\begin{array}{c}\text { Sample } \\
\hat{S E}\end{array}$ & 0.1136 & 0.1580 & -4.7906 & 113.0720 \\
$(0.0460)$ & $(0.0004)$ & $(2.6635)$ & $(53.9954)$ \\
\hline \hline $\begin{array}{c}\text { Full Model } \\
\hat{S E}\end{array}$ & 0.1258 & 0.1568 & -0.6550 & 27.6092 \\
$(0.0434)$ & $(0.0100)$ & $(0.3975)$ & $(9.0859)$ \\
\hline $\begin{array}{c}\text { "pure GARCH" } \\
\hat{S E}\end{array}$ & 0.1596 & 0.1693 & 0 & 6.2298 \\
$(0.0352)$ & $(0.0222)$ & 0 & $(3.1323)$ \\
\hline $\begin{array}{c}\text { "pure Jump" } \\
\hat{S E}\end{array}$ & 0.1136 & 0.1569 & -0.2282 & 13.5363 \\
$(0.0494)$ & $(0.0053)$ & $(0.1221)$ & $(1.7658)$ \\
\hline \hline
\end{tabular}

The Standard Errors of the sample moments are obtained using a GMM approach. The variance-covariance matrix is estimated using a Newey-West Estimator with 5 lags. 